University of Nebraska - Lincoln

DigitalCommons@University of Nebraska - Lincoln

U.S. Department of Veterans Affairs Staff

Publications

U.S. Department of Veterans Affairs

2010

\title{
Gait Variability Patterns are Altered in Healthy Young Individuals During the Acute Reperfusion Phase of Ischemia-Reperfusion
}

Sara A. Myers

University of Nebraska at Omaha, samyers@unomaha.edu

Nikolaos Stergiou

University of Nebraska at Omaha, nstergiou@unomaha.edu

Iraklis I. Pipinos

University of Nebraska Medical Center, ipipinos@unmc.edu

Jason M. Johanning

University of Nebraska Medical Center, jjohanning@unmc.edu

Follow this and additional works at: https://digitalcommons.unl.edu/veterans

Myers, Sara A.; Stergiou, Nikolaos; Pipinos, Iraklis I.; and Johanning, Jason M., "Gait Variability Patterns are Altered in Healthy Young Individuals During the Acute Reperfusion Phase of Ischemia-Reperfusion" (2010). U.S. Department of Veterans Affairs Staff Publications. 56.

https://digitalcommons.unl.edu/veterans/56

This Article is brought to you for free and open access by the U.S. Department of Veterans Affairs at DigitalCommons@University of Nebraska - Lincoln. It has been accepted for inclusion in U.S. Department of Veterans Affairs Staff Publications by an authorized administrator of DigitalCommons@University of Nebraska Lincoln. 


\title{
ASSOCIATION FOR ACADEMIC SURGERY
}

\section{Gait Variability Patterns are Altered in Healthy Young Individuals During the Acute Reperfusion Phase of Ischemia-Reperfusion}

\author{
Sara A. Myers, M.S., ${ }^{*}$ Nick Stergiou, Ph.D., ${ }^{*} \S$ Iraklis I. Pipinos, M.D., $\dagger \ddagger$ and Jason M. Johanning, M.D.† ${ }^{1}$ \\ *Nebraska Biomechanics Core Facility, University of Nebraska at Omaha, Omaha; Nebraska; †Department of Surgery, \\ University of Nebraska Medical Center, Omaha, Nebraska; $\ddagger$ Department of Surgery, Veterans Affairs Medical Center of Nebraska \\ and Western Iowa, Omaha, Nebraska; and §College of Public Health, University of Nebraska Medical Center, Omaha, Nebraska
}

Submitted for publication December 16, 2009

\begin{abstract}
Background. The role of ischemia reperfusion contributing to functional impairment in lower extremity peripheral arterial disease (PAD) patients has not previously been elucidated. The evaluation of gait variability patterns has proven useful in many pathologic populations. Therefore, the purpose of this study is to isolate and determine the specific effect of the acute reperfusion phase of ischemia-reperfusion on gait variability in young individuals with no vascular disease.

Materials and Methods. Thirty healthy young individuals walked on a treadmill during baseline and the acute reperfusion phase of ischemia-reperfusion conditions while lower extremity joint kinematics were captured. Stride to stride variability was assessed using the largest Lyapunov exponent, approximate entropy, standard deviation, and coefficient of variation. Differences in gait variability between conditions were assessed using dependent $t$-tests.
\end{abstract}

Results. The largest Lyapunov exponent values and approximate entropy values were significantly higher in the acute reperfusion phase of ischemiareperfusion condition for the ankle, knee, and the hip. Coefficient of variation was significantly higher at the hip and standard deviation was higher at the knee and the hip during the acute reperfusion phase of ischemia-reperfusion condition.

Conclusions. The acute reperfusion phase of the ischemia-reperfusion cycle alters gait variability patterns at the ankle, knee, and the hip in healthy young individuals. Our findings indicate increased noise and irregularity of gait variability patterns postischemia. In young healthy individuals who do not

\footnotetext{
1 To whom correspondence and reprint requests should be addressed at University of Nebraska Medical Center, 983280 Nebraska Medical Center, Omaha, NE 68198-3280. E-mail:jjohanning@unmc edu.
}

have neuromuscular impairments, significant gait alterations are present during walking after a period of interruption of blood flow. $\odot 2010$ Elsevier Inc. All rights reserved.

Key Words: peripheral arterial disease; atherosclerosis; peripheral vascular disease.

\section{INTRODUCTION}

The differences in gait parameters that occur across multiple steps are referred to as gait variability. The presence of a certain amount and an ordered structure of variability during movement are thought to be "healthy," and these fluctuations allow individuals to adjust to changing stresses encountered during daily activities, including walking. Changes to this "optimal" amount or structure of variability are generally associated with disease and could be related with several physiologic factors such as neural control, muscle function and posture [1]. Increased gait variability has been associated with unsteadiness during walking [2] and increased variability in elderly individuals has been linked to increased risk of falling [3-7]. Previous work in our laboratory determined that symptomatic peripheral arterial disease (PAD) patients have increased lower extremity gait variability compared with healthy matched controls [8]. This is consistent with significant deterioration in the locomotor system of PAD patients. However, the specific mechanisms resulting in this deterioration and leading to mobility problems in patients with PAD remain unclear. Possible mechanisms resulting in functional impairment of PAD patients include insufficient blood flow, underlying neural and muscular abnormalities of the lower extremity, and systemic co-morbidities [9]. To attempt to isolate and determine the impact of reduced flow on gait 
parameters we evaluated joint kinematic variability during baseline and the acute reperfusion phase of ischemia reperfusion in healthy young individuals. We hypothesized that evaluation of the acute reperfusion phase of ischemia-reperfusion in the absence of underlying neuromuscular or systemic dysfunction would result in significant gait variability alterations in comparison with baseline gait.

\section{METHODS}

\section{Participants}

Thirty healthy young participants (age: $22.8 \pm 4.16 \mathrm{y}$, mass: $75.8 \pm$ $13.4 \mathrm{~kg}$, height: $175.3 \pm 8.7 \mathrm{~cm}$, gender: 26 males, 4 females) were recruited to participate in the study from the campus of the University of Nebraska at Omaha. The gender composition was recruited to reflect the composition of the PAD patients based on our previous studies [8, 10-12]. Informed consent was obtained from all participants before data collection according to the guidelines of the Institutional Review Board. Subjects were free from any significant health comorbidities, including arthritis, history of lower extremity joint surgery, history of back or lower extremity injury, or surgery that affects the subject's mobility or any other process limiting the ability to walk, including neurologic disease or impairment (stroke, Parkinson's disease, multiple sclerosis). Additionally, all subjects had normal ankle brachial index values (ABI >1.0), and no subjective or objective ambulatory dysfunction. All subjects reported to our biomechanics facility for gait testing.

\section{Experimental Procedure and Data Collection}

Upon arrival to the laboratory, lower extremity blood flow was measured by taking the systolic pressures at the brachial artery in the arm and the dorsal pedis and posterior tibial arteries at the ankle to confirm acceptable ABI values. Next, subjects' height, weight, and anthropometric measures were taken. Before data collection, reflective markers were placed as previously described [8]. After the markers were placed, participants were given ample time to get accustomed to walking on the treadmill (BodyGuard Fitness; St-Georges, QC, Canada), during which time they were asked to select a comfortable walking speed. This speed was identified as the self-selected walking speed.

Three-dimensional kinematics were acquired at $60 \mathrm{~Hz}$ using EVART software (Motion Analysis Corp., Santa Rosa, CA) while participants walked on the treadmill. First the participants walked at their self-selected speed for $3 \mathrm{~min}$. This was the baseline condition. Next, vascular occlusion was induced by placing thigh cuffs (Omron, Kyoto, Japan, Exactus Aneroid Sphygmomanometer model 108MLNL) bilaterally on the upper thighs and occlusion tourniquets (CyberTech, Maharashtra, India, Mechanical Advantage Tourniquet MAT01) just above the knee while subjects stood on the treadmill. The cuffs were inflated to $200 \mathrm{mmHg}$ and maintained for $3 \mathrm{~min}$. The chosen level of pressure and time of occlusion are standard ranges used in the literature to induce ischemia in the legs [13-18]. After 3 min of occlusion, the thigh cuffs were removed and the subjects immediately began walking on the treadmill. Three minutes of treadmill walking was recorded during the acute reperfusion phase of ischemia reperfusion.

\section{Data Analysis}

Coordinate trajectories of each marker were exported and processed in custom software using MATLAB software (MathWorks Inc., Natick, MA). This software was used to calculate relative joint angle time series from the kinematic data for the ankle, knee, and hip for all trials. Joint kinematic variability was examined, because it has been shown that variability of stride characteristics (i.e., stride time, step time) offers a less sensitive measure of differences between groups than does variability of the joint kinematics [19]. All trials were cropped to 3300 data points, which corresponds to precisely 55 $\mathrm{s}$ and is long enough to allow 30 continuous footfalls which is considered adequate for nonlinear analysis [20,21]. The data were analyzed unfiltered to achieve the most accurate representation of the variability within the locomotor system. The same data collection system was used for all participants and, therefore, we assumed the level of measurement noise was consistent between subjects. Thus, any differences between conditions can be recognized as differences in the locomotor system during the acute reperfusion phase of ischemia reperfusion.

\section{Linear Analysis}

The linear analysis provides information about the amount of variability present in the gait patterns and is used to complement the nonlinear analysis. Range of motion of the ankle, knee, and hip angles were calculated for each gait cycle and for every time series. Means, standard deviations, and coefficients of variation were then calculated for each variable and for each participant. MATLAB software was used for these calculations.

\section{Nonlinear Analysis}

Nonlinear analysis methods included in the analysis were the largest Lyapunov exponent and approximate entropy. Unlike the linear statistics, both the largest Lyapunov exponent and approximate entropy take into account the entire time series of the joint angle, rather than looking at a few specific points in the series [20]. The largest Lyapunov exponent is a measure of the rate of divergence of neighbored state-space trajectories, and it estimates the sensitivity of the locomotor system to perturbations. The largest Lyapunov exponent quantifies the exponential separation of nearby trajectories in the reconstructed state space of the joint angle time series. As nearby points of the state space separate, they diverge rapidly and can produce instability. The largest Lyapunov exponent from a stable system with little to no divergence will be zero (e.g., sine wave). Alternatively, the largest Lyapunov exponent for an unstable system that has a high amount of divergence will be positive with a larger value $(>0.5 ;$ Fig. 1$)$ [7, 20, 22]. The Chaos Data Analyzer professional version (American Institute of Physics [23]) was used to numerically calculate the largest Lyapunov exponent for each joint angle time series for each participant. Refer to Appendix A for a detailed description of the actual calculation of this measure.

A method to determine the complexity in the gait time series is to compute the approximate entropy [20, 24, 25]. Approximate entropy is a measure that can quantify the regularity or predictability of a time series [26]. A more predictable and regular time series is also less complex. A change in complexity may be indicative of learning and a reorganization of the available degrees of freedom [26, 27]. The approximate entropy measures the logarithmic probability that a series of data points a certain distance apart will exhibit similar relative characteristics on the next incremental comparison with the state space [27-29]. Time series with a greater likelihood of remaining the same distance apart upon comparison will result in lower approximate entropy values, while data points that exhibit large differences in distances between data points will result in higher values. Values typically range from zero to two. Values closer to zero are consistent with greater periodicity (less complexity). Conversely, values nearing two represent greater irregularity (higher complexity). The approximate entropy value for a periodic time series such as the sine wave will be close to zero, for a random signal such as white noise will be close to two, while a deterministic signal like the joint angle time series will be 
A
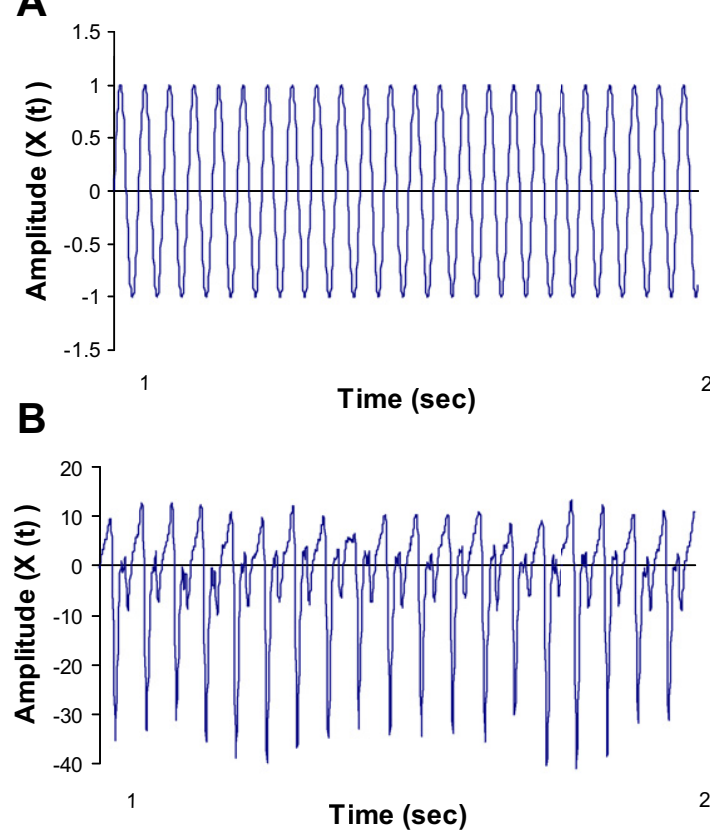

C

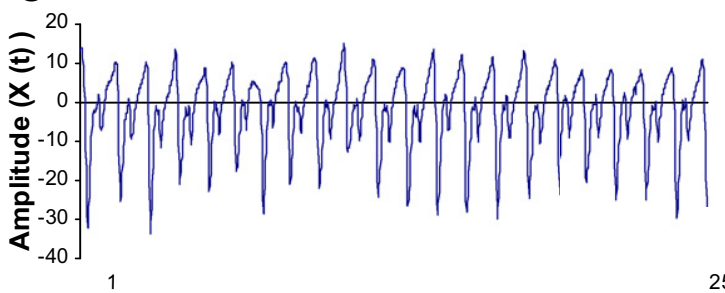

D

Time (sec)

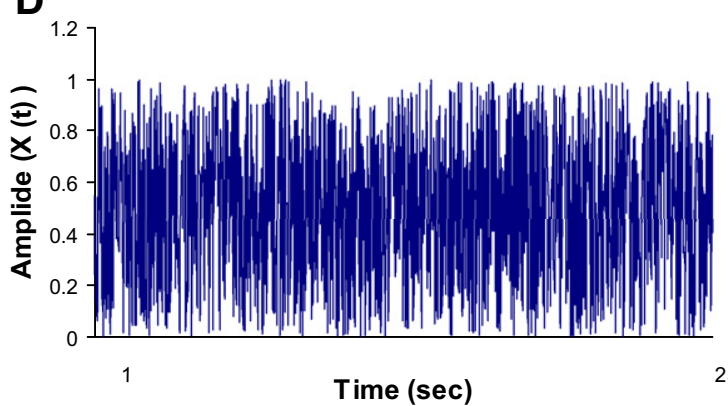

25
E 0.002

0.0015

0.001

$\approx^{0.0005}$

$\times$

$-0.001$

$-0.0015$

$-0.002$

$\mathbf{F}$

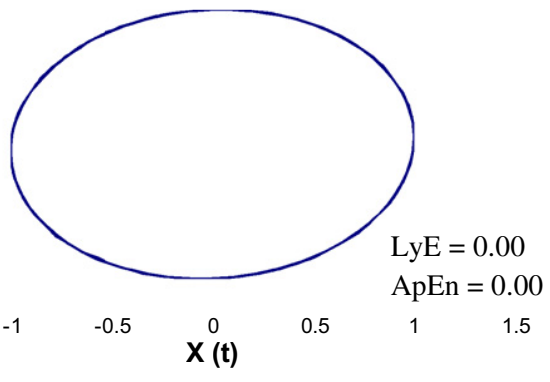

0.1

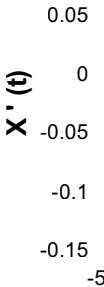

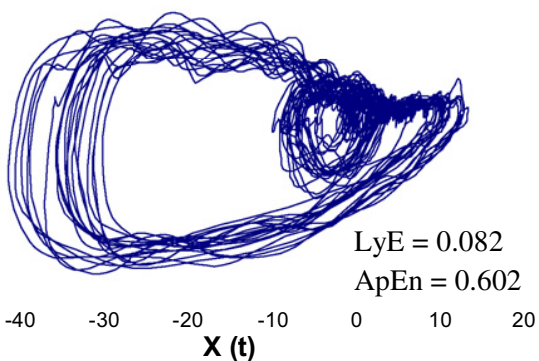

G 0.1

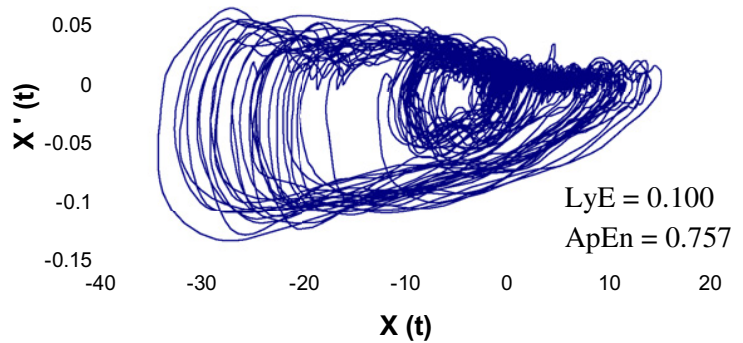

H

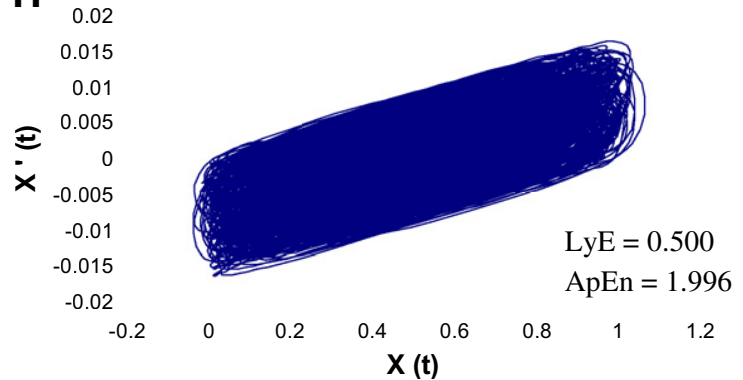

FIG. 1. A visual comparison of variability between a (A) known periodic signal (sine wave), (B) ankle joint flexion-extension from a representative subject during baseline walking, (C) ankle joint flexion-extension from the same representative subject during walking in the acute reperfusion phase of ischemia-reperfusion, and (D) known random signal (white noise). The two-dimensional state spaces in graphs (E), (F), (G), and $(\mathrm{H})$ were created by plotting the position $[\mathrm{X}(\mathrm{t})]$ versus the velocity $\left[\mathrm{X}^{\prime}(\mathrm{t})\right]$ from the corresponding signals. The calculated largest Lyapunov exponent ( $\mathrm{LyE})$ and approximate entropy ( $\mathrm{ApEn}$ ) values are shown for each signal. (Color version of figure is available online.)

somewhere in between (Fig. 1). A detailed description of the calculation of the approximate entropy can be found in Appendix B.

When using nonlinear analysis techniques, it is important to validate results against surrogate data to distinguish a deterministic origin from randomness. Surrogation is also an important measure used to determine if the source of the variation is deterministic in nature [7, $20,30]$. This method compares the original time series data set and an equivalent random data set with similar structure. Surrogation removes the deterministic characteristics from the actual data set, leaving a random series with the same mean, variance and power spectra as the original data. Significant differences between LyE values for the original and surrogate time series indicate that the variations observed in the actual data series are not random in nature and have deterministic properties [20]. Surrogation analysis was performed on every continuous joint angle using the method described by Small et al. [30, 31] for periodic time series.

\section{Statistical Analysis}

Means for the standard deviation and the coefficient of variation of the range of motion, largest Lyapunov exponent values, and 
approximate entropy values were calculated for the ankle, knee, and hip joints for the baseline and acute reperfusion phase of ischemiareperfusion conditions. Differences between conditions were determined using dependent $t$-tests. To ensure that the sample of differences are normally distributed and confirm the applicability of the dependent $t$-tests, the Shapiro-Wilk test for normality was calculated for each dependent variable. The average $\mathrm{W}$ values suggest that our data is from a normal distribution (Table 1). Statistical comparisons were performed using SPSS 12.0 software (SPSS Inc., Chicago, IL) with a level of significance set at $\alpha=0.05$.

\section{RESULTS}

There was a significant increase in standard deviation of the knee and hip joint range of motion in the acute reperfusion phase of ischemia-reperfusion condition (Table 1). Additionally, there was a significant increase in the coefficient of variation of the hip joint range of motion during the acute reperfusion phase of ischemiareperfusion condition as compared with the baseline condition. These differences indicate an increase in the amount of variability at the hip and the knee during the acute reperfusion phase of ischemia reperfusion.

Regarding structure of variability, there were significant differences between baseline and acute reperfusion phase of ischemia-reperfusion conditions for the ankle, knee, and hip joints time series (Table 1). Specifically, the largest Lyapunov exponent and approximate entropy values increased post vascular occlusion. These increases indicate a change in the structure of variability while walking in the acute reperfusion phase of ischemia-reperfusion. Increases in the largest Lyapunov exponent show that joint movement patterns were farther apart in consecutive strides. For the ap- proximate entropy, larger values represent greater irregularity of the joint angle time series. For the largest Lyapunov exponent and the approximate entropy, the mean difference between conditions progressively increased in the more distal joints.

For the surrogation analysis, the surrogate data series had significantly higher largest Lyapunov exponent values than the original data for the ankle, knee, and the hip during walking in both the baseline and the acute reperfusion phase of ischemia-reperfusion conditions (Table 1).

\section{DISCUSSION}

The purpose of this study was to isolate and determine the specific effect of the acute reperfusion phase of ischemia-reperfusion on gait variability in individuals without the neuromuscular and systemic impairments that exist in patients with PAD. We assessed this by inducing lower extremity vascular occlusion and examining gait variability by evaluating the joint kinematics of the lower extremities in healthy young individuals. Gait variability was chosen because it is associated with risk of falling, and previously found in our laboratory to be altered in patients with PAD [8]. We hypothesized that the gait of healthy young individuals would be altered during the acute reperfusion phase of ischemia-reperfusion.

Nonlinear analysis demonstrated significant gait variability changes for all lower extremity joints based on the largest Lyapunov exponent and approximate entropy. For the linear measures, differences were seen

TABLE 1

Mean values for the largest Lyapunov exponent of the original time series (LyE) and the surrogate time series (LyE-S), approximate entropy (ApEn), standard deviation (SD) and coefficient of variation (CoV) for healthy young participants in the baseline and acute reperfusion phase of ischemia-reperfusion (Post) conditions

\begin{tabular}{|c|c|c|c|c|c|c|c|c|c|}
\hline Condition $(n=30)$ & Ankle & $P$ value & $W$ & Knee & $P$ value & $W$ & Hip & $P$ value & $W$ \\
\hline \multicolumn{10}{|l|}{ LyE } \\
\hline Baseline & $0.069 \pm 0.02^{*}$ & \multirow[t]{2}{*}{$P=0.043$} & 0.953 & $0.066 \pm 0.02 *$ & \multirow[t]{2}{*}{$P=0.002$} & 0.947 & $0.066 \pm 0.02^{*}$ & \multirow[t]{2}{*}{$P=0.040$} & 0.963 \\
\hline Post & $0.088 \pm 0.02$ & & 0.975 & $0.081 \pm 0.02$ & & 0.975 & $0.076 \pm 0.02$ & & 0.967 \\
\hline \multicolumn{10}{|l|}{ LyE-S } \\
\hline Baseline & $0.089 \pm 0.02^{+}$ & $P<0.001$ & 0.955 & $0.084 \pm 0.02^{+}$ & $P<0.001$ & 0.974 & $0.080 \pm 0.02^{+}$ & $P<0.001$ & 0.978 \\
\hline Post & $0.094 \pm 0.02^{+}$ & $P<0.001$ & 0.950 & $0.096 \pm 0.03^{+}$ & $P<0.001$ & 0.841 & $0.088 \pm 0.03^{+}$ & $P=0.010$ & 0.731 \\
\hline \multicolumn{10}{|l|}{ ApEn } \\
\hline Baseline & $0.712 \pm 0.13^{*}$ & \multirow[t]{2}{*}{$P<0.001$} & 0.960 & $0.431 \pm 0.08^{*}$ & \multirow[t]{2}{*}{$P<0.001$} & 0.961 & $0.307 \pm 0.06^{*}$ & \multirow[t]{2}{*}{$P<0.001$} & 0.953 \\
\hline Post & $0.858 \pm 0.17$ & & 0.921 & $0.504 \pm 0.08$ & & 0.946 & $0.374 \pm 0.08$ & & 0.954 \\
\hline \multicolumn{10}{|l|}{ SD } \\
\hline Baseline & $3.09 \pm 1.30$ & \multirow[t]{2}{*}{$P=0.399$} & 0.916 & $1.36 \pm 0.43^{*}$ & \multirow[t]{2}{*}{$P=0.001$} & 0.976 & $1.17 \pm 0.37 *$ & \multirow[t]{2}{*}{$P=0.001$} & 0.889 \\
\hline Post & $3.34 \pm 1.32$ & & 0.913 & $1.91 \pm 0.71$ & & 0.902 & $1.52 \pm 0.54$ & & 0.921 \\
\hline \multicolumn{10}{|l|}{$\mathrm{CoV}$} \\
\hline Baseline & $9.07 \pm 4.24$ & \multirow{2}{*}{$P=0.112$} & 0.900 & $2.51 \pm 0.99$ & \multirow[t]{2}{*}{$P=0.271$} & 0.948 & $3.60 \pm 1.46^{*}$ & \multirow[t]{2}{*}{$P=0.005$} & 0.846 \\
\hline Post & $10.83 \pm 4.15$ & & 0.976 & $2.86 \pm 1.56$ & & 0.789 & $4.84 \pm 1.90$ & & 0.886 \\
\hline
\end{tabular}

Data are reported as mean \pm SD. Significant differences $(\mathrm{P}<0.05)$ between baseline and post conditions are marked with an asterisk $(*)$. Significant differences between the original time series and their surrogate counterparts are marked with a superscript plus sign $\left(^{+}\right)$. $W$ indicates statistic value from the Shapiro-Wilks test for normality. 
only for the knee and the hip. Therefore, our hypothesis of altered variability was supported and, more specifically, the differences indicated an increase in the noise, randomness, and instability of the locomotor system and an increase in the amount of variability while walking during the acute reperfusion phase of ischemiareperfusion.

In the current study, the healthy young subjects' baseline condition is considered to have the "optimal" amount of variability. The changes to gait variability between baseline and the acute reperfusion phase of ischemia-reperfusion conditions in healthy individuals are similar to those found in a previous study between healthy matched controls and patients with PAD [8]. Utilizing data from our previous studies, we can make direct comparisons between the response of young healthy subjects and PAD patients [8]. For this comparison, we consider the average differences from the young baseline condition expressed as percentage change averaged across the ankle, knee, and the hip for the acute reperfusion phase of ischemia-reperfusion condition and for PAD patients compared with the young baseline condition. The acute reperfusion phase of ischemia-reperfusion condition had average increases of $21 \%$ for the largest Lyapunov exponent, $26 \%$ for the standard deviation, and of $22 \%$ for the coefficient of variation as compared with the baseline condition. Comparing variability values from a previous investigation of $\mathrm{PAD}$ patients [8], $\mathrm{PAD}$ patients on average had increases of $48 \%$ for largest Lyapunov exponent values, $62 \%$ for the standard deviation, and $99 \%$ for the coefficient of variation. The acute reperfusion phase of ischemia-reperfusion condition did lead to increases in the largest Lyapunov exponent, standard deviation, and coefficient of variation, but the presence of PAD led to even greater increases. The difference in gait variability from baseline to the acute reperfusion phase of ischemia-reperfusion condition in healthy young is clearly the result of ischemia-reperfusion that resulted from the induced vascular occlusion procedure in this study. However, the ischemiareperfusion condition does not alter gait variability to the same magnitude as $\mathrm{PAD}$, which suggests that other manifestations of the pathophysiology of PAD are leading to additional differences. These additional increases in variability seen in PAD patients are likely due to underlying cellular abnormalities in the lower extremity muscles and nerves that have been demonstrated in these patients [32-35].

In previous biomechanical studies of gait in $\mathrm{PAD}$ patients, changes have been consistently documented at the ankle, with differences in kinematics [12, 36], kinematic variability [8], and peak plantar flexion torque at late stance [11] between PAD patients and healthy matched controls. The fact that gait variability changes in the acute reperfusion phase were pronounced at the ankle in healthy young individuals demonstrates that the calf musculature appears to be the end organ of ischemia with regards to gait. This finding is consistent with the significant changes occurring in the lower extremities in patients with PAD [37-39]. The question remains, however, on how quickly the neuromuscular changes occur and over what time period these changes take place.

Results from the surrogation analysis established that the largest Lyapunov exponent values of the original time series were significantly different from their surrogate counterparts for the ankle, knee, and the hip during both conditions (baseline and acute reperfusion phase of ischemia-reperfusion). Surrogation analysis was performed to confirm that our data has a deterministic origin (the time series is not random). Our findings demonstrate that the variability present in the subjects is deterministic, even in the acute reperfusion phase of ischemia-reperfusion. Significant differences between original and surrogate time series were previously found in healthy young and elderly populations during normal walking conditions $[7,30]$. The presence of determinism in the variability of gait patterns provides individuals with the ability to respond to challenging circumstances that may affect walking conditions (i.e., icy sidewalks, walking in crowds). Even though the gait variability had increased noise in the acute reperfusion phase of ischemia-reperfusion, the deterministic structure of variability was maintained. This finding is in contrast to results of surrogation analysis in PAD patients that showed a degradation of the variability structure during pain free walking [8]. Therefore, while the deterministic structure was degraded with PAD, acute perturbations, such as the acute reperfusion phase of ischemia-reperfusion used in the current study, does not impact the neuromuscular system of healthy young individuals to the same extent.

Although the ischemia was produced by occlusion in the proximal thigh distal to the hip joint, significant differences in gait function were noted for the hip joint. Gait differences at the hip could be an attempt to compensate for ankle and knee alterations. Conversely, ischemia within the quadriceps and hamstrings may have contributed to the alterations of the hip joint. Regardless, our data would suggest that the level of disease and distribution of ischemia may play a significant role in the gait patterns in $\mathrm{PAD}$ patients. Based on the results of this investigation, muscles proximal to the atherosclerotic blockage may also be affected. Future research is needed to examine the effect of level of disease on functional impairments in patients with PAD.

A limitation of the study is that it is difficult to specifically isolate the effect of reduced blood flow, especially 
because using a tourniquet creates obstruction to venous outflow in addition to reduced arterial inflow. While it is not possible to continuously monitor the ankle-brachial index of subjects, the current study created an acute period of clinical ischemia consistent with reduced arterial inflow. The experimental design is similar to the vascular laboratory technique of ischemia-reperfusion, which creates a supply-demand imbalance and creates a low grade ischemia of the distal muscle beds. In contrast to the PAD patients, reperfusion in healthy young individuals likely occurs quickly. Despite these factors, ambulatory functions during the acute reperfusion phase of ischemiareperfusion were significantly changed. Obviously, the ideal experiment would be temporary occlusion of the arterial circulation in an invasive manner, which for obvious reasons is not feasible in normal healthy subjects.

\section{CONCLUSIONS}

Results of our study indicate that ischemiareperfusion, in the absence of neuromuscular and systemic pathology and comorbidities, significantly alters gait variability patterns. This study demonstrates that ischemia-reperfusion significantly alters gait in healthy young individuals. However, the change in the gait variability patterns was not as severe as previously documented in symptomatic PAD patients during pain free ambulation. Therefore, the current study demonstrates that gait variability differences are present during the acute reperfusion phase of ischemia reperfusion, however, our results also support the hypothesis promoted by our group that damaged muscles and nerves in the lower extremities further contribute to altered gait variability patterns in patients with PAD.

\section{ACKNOWLEDGMENTS}

This work was supported by funds received from the American Geriatrics Society's Hartford Foundation Dennis W. Jahnigen Award (to JMJ), the Nebraska Research Initiative (to NS), the American Society of Biomechanics, the American Alliance for Health, Physical Education, Recreation, and Dance Research Grant program, National Institute On Aging (Award Number F31AG032788), and the NASA Nebraska Space Grant Fellowship (to SM).

\section{SUPPLEMENTARY DATA}

Supplementary data associated with this article can be found, in the online version, at doi:10.1016/j.jss. 2010.04.030.

\section{REFERENCES}

1. Stergiou N, Harbourne R, Cavanaugh J. Optimal movement variability: A new theoretical perspective for neurologic physical therapy. J Neurol Phys Ther 2006;30:120.

2. Brach JS, Berlin JE, Van Swearingen JM, et al. Too much or too little step width variability is associated with a fall history in older persons who walk at or near normal gait speed. J Neuroeng Rehabil 2005;26:21.

3. Hausdorff JM, Edelberg HK, Mitchell SL, et al. Increased gait unsteadiness in community-dwelling elderly fallers. Arch Phys Med Rehabil 1997;78:278.

4. Hausdorff JM, Edelberg HK, Cudkowicz ME, et al. The relationship between gait changes and falls. J Am Geriatr Soc 1997; 45:1406.

5. Kurz MJ, Stergiou N. The aging human neuromuscular system expresses less certainty for selecting joint kinematics during gait. Neurosci Lett 2003;348:155.

6. Maki BE. Gait changes in older adults: Predictors of falls or indicators of fear. J Am Geriatr Soc 1997;45:313.

7. Buzzi UH, Stergiou N, Kurz MJ, et al. Nonlinear dynamics indicates aging affects variability during gait. Clin Biomech (Bristol, Avon) 2003;18:435.

8. Myers SA, Johanning JM, Stergiou N, et al. Gait variability is altered in patients with peripheral arterial disease. J Vasc Surg 2009;49:924.e1.

9. McDermott MM, Greenland P, Liu K, et al. Leg symptoms in peripheral arterial disease: Associated clinical characteristics and functional impairment. JAMA 2001;286:1599.

10. Scott-Pandorf MM, Stergiou N, Johanning JM, et al. Peripheral arterial disease affects ground reaction forces during walking. J Vasc Surg 2007;46:491.

11. Chen SJ, Pipinos II, Johanning JM, et al. Bilateral intermittent claudication results in alterations in the gait biomechanics at the hip and ankle joints during gait. J Biomech 2008;41:2506.

12. Celis R, Pipinos II, Scott-PandorfMM, et al. Peripheral arterial disease affects kinematics during walking. J Vasc Surg 2009;:49127.

13. Diener HC, Dichgans J, Guschlbauer B, et al. The significance of proprioception on postural stabilization as assessed by ischemia. Brain Res 1984;296:103.

14. Kjaer M, Pott F, Mohr T, et al. Heart rate during exercise with leg vascular occlusion in spinal cord-injured humans. J Appl Physiol 1999;86:806.

15. Sargeant AJ, Rouleau MY, Sutton JR, et al. Ventilation in exercise studied with circulatory occlusion. J Appl Physiol 1981; 50:718.

16. Stanley WC, Lee WR, Brooks GA. Ventilation studied with circulatory occlusion during two intensities of exercise. Eur J Appl Physiol Occup Physiol 1985;54:269.

17. Haouzi P, Huszczuk A, Porszasz J, et al. Femoral vascular occlusion and ventilation during recovery from heavy exercise. Respir Physiol 1993;94:137.

18. Tokizawa K, Mizuno M, Nakamura Y, et al. Venous occlusion to the lower limb attenuates vasoconstriction in the nonexercised limb during post-handgrip muscle ischemia. J Appl Physiol 2004;96:981.

19. Barrett R, Noordegraaf MV, Morrison S. Gender differences in the variability of lower extremity kinematics during treadmill locomotion. J Mot Behav 2008;40:62.

20. Stergiou N, Buzzi UH, Kurz MJ, et al. Nonlinear tools in human movement. In: Stergiou N, Ed. Innovative analysis of human movement. Champaign, IL: Human Kinetics, 2004:63-90.

21. Keenan S, Stergiou N. The reliability of the Lyapunov exponent during treadmill walking. Proceedings of the 27th Annual Meeting of the American Society of Biomechanics; Toledo, OH, 2002.

22. Abarbanel HDI. Analysis of observed chaotic data. New York: Springer-Verlag, 1996.

23. Sprott J, Rowlands G. Chaos data analyzer: The professional version. 1992.

24. Pincus SM, Goldberger AL. Physiological time-series analysis: What does regularity quantify? Am J Physiol 1994;266:H1643.

25. Georgoulis AD, Moraiti C, Ristanis S, et al. A novel approach to measure variability in the anterior cruciate ligament deficient knee during walking: The use of the approximate entropy in orthopaedics. J Clin Monit Comput 2006;20:11. 
26. Pincus SM, Gladstone IM, Ehrenkranz RA. A regularity statistic for medical data analysis. J Clin Monit 1991;7:335.

27. Pincus SM. Approximate entropy (ApEn) as a complexity measure. Chaos 1995;5:110.

28. Pincus SM. Approximate entropy (ApEn) as a regularity measure. In: Newell KM, Molenaar PCM, Eds. Applications of nonlinear dynamics to developmental process modeling. Mahwah, NJ: Lawrence Erlbaum Associates 1997:243-68.

29. Pincus SM. Irregularity and asynchrony in biologic network signals. Methods Enzymol 2000;321:149.

30. Miller DJ, Stergiou N, Kurz MJ. An improved surrogate method for detecting the presence of chaos in gait. J Biomech 2006; 39:2873.

31. Small M, Yu D, Harrison RG. Surrogate test for pseudoperiodic time series data. Phys Rev Lett. 2001 10/16/;87(18):188101. Available at: http://link.aps.org/abstract/PRL/v87/e188101. Accessed June 15, 2007.

32. Brass EP, Hiatt WR. Acquired skeletal muscle metabolic myopathy in atherosclerotic peripheral arterial disease. Vasc Med 2000;5:55.

33. Pipinos II, Boska MD, Shepard AD, et al. Pentoxifylline reverses oxidative mitochondrial defect in claudicating skeletal muscle. J Surg Res 2002;102:126.
34. Pipinos II, Judge AR, Zhu Z, et al. Mitochondrial defects and oxidative damage in patients with peripheral arterial disease. Free Radic Biol Med 2006;41:262.

35. Pipinos II, Shepard AD, Anagnostopoulos PV, et al. Phosphorus 31 nuclear magnetic resonance spectroscopy suggests a mitochondrial defect in claudicating skeletal muscle. J Vasc Surg 2000;31:944.

36. Crowther RG, Spinks WL, Leicht AS, et al. Relationship between temporal-spatial gait parameters, gait kinematics, walking performance, exercise capacity, and physical activity level in peripheral arterial disease. J Vasc Surg 2007;45:1172.

37. Pipinos II, Judge AR, Selsby JT, et al. The myopathy of peripheral arterial occlusive disease: Part 2. oxidative stress, neuropathy, and shift in muscle fiber type. Vasc Endovasc Surg 2008; 42:101.

38. Pipinos II, Swanson SA, Zhu Z, et al. Chronically ischemic mouse skeletal muscle exhibits myopathy in association with mitochondrial dysfunction and oxidative damage. Am J Physiol Regul Integr Comp Physiol 2008;295:R290.

39. Pipinos II, Judge AR, Selsby JT, et al. The myopathy of peripheral arterial occlusive disease: Part 1. Functional and histomorphologic changes and evidence for mitochondrial dysfunction. Vasc Endovasc Surg 2007;41:481. 